\title{
Animal experimental research design in critical care
}

\author{
Justin S. Merkow ${ }^{1}$, Janine M. Hoerauf ${ }^{1}$, Angela F. Moss², Jason Brainard', Lena M. Mayes ${ }^{1}$, \\ Ana Fernandez-Bustamante', Susan K. Mikulich-Gilbertson ${ }^{3,4}$ and Karsten Bartels ${ }^{1 *}$
}

\begin{abstract}
Background: Limited translational success in critical care medicine is thought to be in part due to inadequate methodology, study design, and reporting in preclinical studies. The purpose of this study was to compare reporting of core features of experimental rigor: blinding, randomization, and power calculations in critical care medicine animal experimental research. We hypothesized that these study design characteristics were more frequently reported in 2015 versus 2005.

Methods: We performed an observational bibliometric study to grade manuscripts on blinding, randomization, and power calculations. Chi-square tests and logistic regression were used for analysis. Inter-rater agreement was assessed using kappa and Gwet's AC1.

Results: A total of 825 articles from seven journals were included. In 2005, power estimations were reported in 2\%, randomization in $35 \%$, and blinding in $20 \%(n=482)$. In 2015, these metrics were included in 9,47 , and $36 \%$ of articles $(n=343)$. The increase in proportion for the metrics tested was statistically significant $(p<0.001, p=0.002$, and $p<0.001)$.

Conclusions: Only a minority of published manuscripts in critical care medicine journals reported on recommended study design steps to increase rigor. Routine justification for the presence or absence of blinding, randomization, and power calculations should be considered to better enable readers to assess potential sources of bias.
\end{abstract}

Keywords: Critical care, Research, Experiment, Study design, Methods

\section{Background}

Despite a significant increase in the volume of biomedical research over the past decade, there has been limited translational success in clinical medicine [1,2]. Reproducibility specifically for animal research is low [3-5]. In attempts to address this problem, the Animal Research: Reporting of In Vivo Experiments (ARRIVE) guidelines as well as the revised National Institutes of Health grant application process have proposed standards for research involving animals to enhance the quality of experimental design, study conduct, and analysis of results [6-8]. These steps are intended to reduce bias and ultimately improve reproducibility and facilitate the translation of biomedical research to novel clinical applications that improve patient outcomes. Additionally,

\footnotetext{
* Correspondence: karsten.bartels@ucdenver.edu

${ }^{1}$ Department of Anesthesiology, Medicine, and Surgery, University of

Colorado, School of Medicine, Anschutz Medical Campus, 12401 E. 17th Ave., Leprino Office Building, 7th Floor, MS B-113, Aurora, CO 80045, USA

Full list of author information is available at the end of the article
}

there is an ethical dilemma regarding animal welfare as well as financial waste related to permitting investment into research without tangible returns [9]. Specifically for the field of critical care medicine, small studies have shown that animal research methodology, study design, and reporting tends to lack rigor in several important areas [10-13].

Improvements in reporting of key experimental design features could enable readers to better judge sources of bias and eventually enhance validity and likelihood of translation. The objective of our study was to evaluate all critical care journals and compare reported animal experimental research in 2005 vs. 2015 regarding power analysis, randomization, and blinding procedures. Our hypothesis was that there had been increased implementation of these methods in 2015 compared to 2005. Also, we sought to provide information on the status quo of reported experimental design features to promote rigor.

(c) The Author(s). 2018 Open Access This article is distributed under the terms of the Creative Commons Attribution 4.0 International License (http://creativecommons.org/licenses/by/4.0/), which permits unrestricted use, distribution, and reproduction in any medium, provided you give appropriate credit to the original author(s) and the source, provide a link to the Creative Commons license, and indicate if changes were made. The Creative Commons Public Domain Dedication waiver (http://creativecommons.org/publicdomain/zero/1.0/) applies to the data made available in this article, unless otherwise stated. 


\section{Methods}

We performed an observational bibliometric analysis of animal research published in critical care medicine journals using PRISMA and STROBE guidelines [14, 15]. Journals were selected based on their inclusion on the Thomson Reuters ${ }^{\mathrm{TM}}$ Journal Citation Reports ${ }^{\circ}$ subject category "Critical Care Medicine" [16]. A PubMed search included animal experimental studies published in 2005 and 2015. Our primary search criterion was that the article was reporting on an animal study based on an experiment. Animals were further defined as: "any of a kingdom of living things composed of many cells typically differing from plants in capacity for active movement, in rapid response to stimulation, in being unable to carry photosynthesis, and lack of cellulose cell walls" [17]. We excluded meta-analyses, case reports, historical articles, letters, review articles, and editorials. One investigator manually assessed the PubMed search results for animal experimental studies. Then, the PubMed filter "other animals" was applied to the initial search results to detect any animal experimental studies not found in the manual search. Journals that did not publish at least ten animal studies in both 2005 and 2015 were excluded from the analysis (Fig. 1). To assess consistency in the identification of manuscripts reporting on animal experimental research, a second investigator blinded to the results of the first investigator independently searched two journals that were randomly selected from the seven journals included in this study.

Next, we rated all animal studies selected. A computer -generated randomization scheme was used to randomize articles by both year and journal before the analysis (Excel, Microsoft Co., Redmond, WA). Studies were analyzed using their full-text Portable Document Format (PDF). Reporting of power analysis, randomization, and blinding was then graded using a $0-3$ point scale (0-not mentioned, 1-mentioned but specified as not performed, 2performed but no details given, 3-performed and details given) [18]. To assess inter-rater agreement for criterion ratings, we randomly selected $10 \%$ of the total articles for

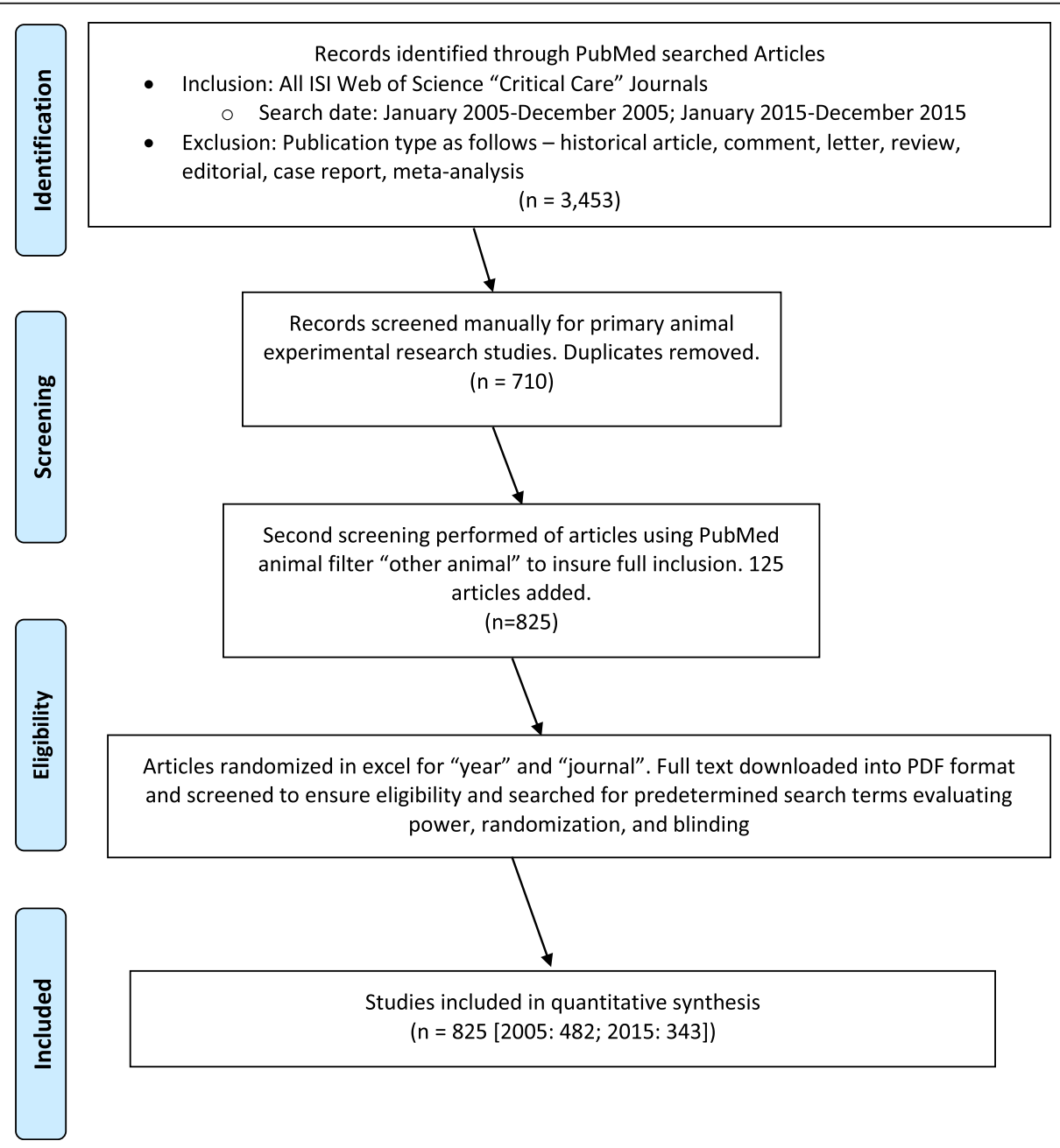

Fig. 1 Study flow diagram 
re-rating by a second investigator blinded to the results of the first investigator.

\section{Statistical analysis}

To address the primary hypothesis, ordinal scale rating scores were collapsed into binary (performed/not performed) variables. Chi-square tests were used to examine overall trends in reporting of quality metrics for 2005 and 2015. Simple logistic regression with time as a continuous covariate was used to estimate the effect of time on quality metrics performed and reported in published articles. The reference group was "not performed", and odds ratios were calculated for the entire 10-year increment in time.

To assess the relationship between year of study and degree of reporting of quality metrics (as ordinal variables), the Wilcoxon Rank Sum test was used. Proportional odds models for ordinal logistic regression was used to calculate an odds ratio for the increase in reporting of metrics in 2015 compared to 2005 . The proportional odds assumptions were verified by the Score Test.

Inter-rater agreement was assessed for each of the three metrics (power, randomization, and blinding) using the Cohen's Kappa and Gwet's AC1 [19]. Gwet's AC1 is an alternative inter-rater reliability coefficient to Cohen's kappa that is more stable in the presence of high prevalence and unbalanced marginal probability $[19,20]$. Inter-rater agreement for identification of animal study articles was assessed using the kappa coefficient. The level of agreement was interpreted using the scale for interpretation of Kappa [21]. The statistical analysis was done in SAS 9.4 (SAS Institute, Cary, NC). Statistical tests were performed adjusting for multiple comparisons using the Bonferroni method to maintain an overall 0.05 level of significance.

\section{Power analysis}

For the power analysis, we assumed a $12 \%$ absolute increase in reporting incidences for each of the three metrics over a 10-year interval in two independent proportions [18]. We anticipated a baseline reporting level of 5\% in 2005 and a reporting level of $17 \%$ in 2015. A total of 141 studies in each year (282 total) would yield $80 \%$ power to detect an absolute difference in the proportion of metrics identified of at least $12 \%$ as significant.

For the randomization metric, we assumed a $13 \%$ absolute increase in reporting incidences for each of the three metrics over a 10-year interval in two independent proportions [18]. We anticipated a baseline reporting level of $41 \%$ in 2005 and a reporting level of $54 \%$ in 2015. A total of 307 studies in each year (614 total) would yield $80 \%$ power to detect an absolute difference in the proportion of metrics identified of at least $13 \%$ as significant.
For the blinding metric, we assumed a $21 \%$ absolute increase in reporting incidences for each of the three metrics over a 10-year interval in two independent proportions [18]. We anticipated a baseline reporting level of $26 \%$ in 2005 and a reporting level of $47 \%$ in 2015. A total of 109 studies in each year (218 total) would yield $80 \%$ power to detect an absolute difference in the proportion of metrics identified of at least $12 \%$ as significant.

All power calculations were done using G*Power, version 3.1.9.2. To maintain a 0.05 significance level across the three outcome metrics, the Bonferroni method for multiple comparisons was used to adjust the alpha to 0.017 .

\section{Results}

After excluding critical care journals that did not publish at least ten animal studies in each year, seven journals comprising 825 articles (482 in 2005, 343 in 2015) were included in the analysis. They included: American Journal of Respiratory and Critical Care Medicine, Burns, Critical Care, Critical Care Medicine, Journal of Neurotrauma, Resuscitation, and Shock. The odds of any of the three metrics being performed in 2015 were higher than in 2005. The breakdown of the changes in reporting frequencies for each journal is depicted in Fig. 2. For power analysis, the odds were 4.52 times $(1.86,11.0)$ higher, for randomization 1.64 times $(1.16,2.31)$ higher, and for

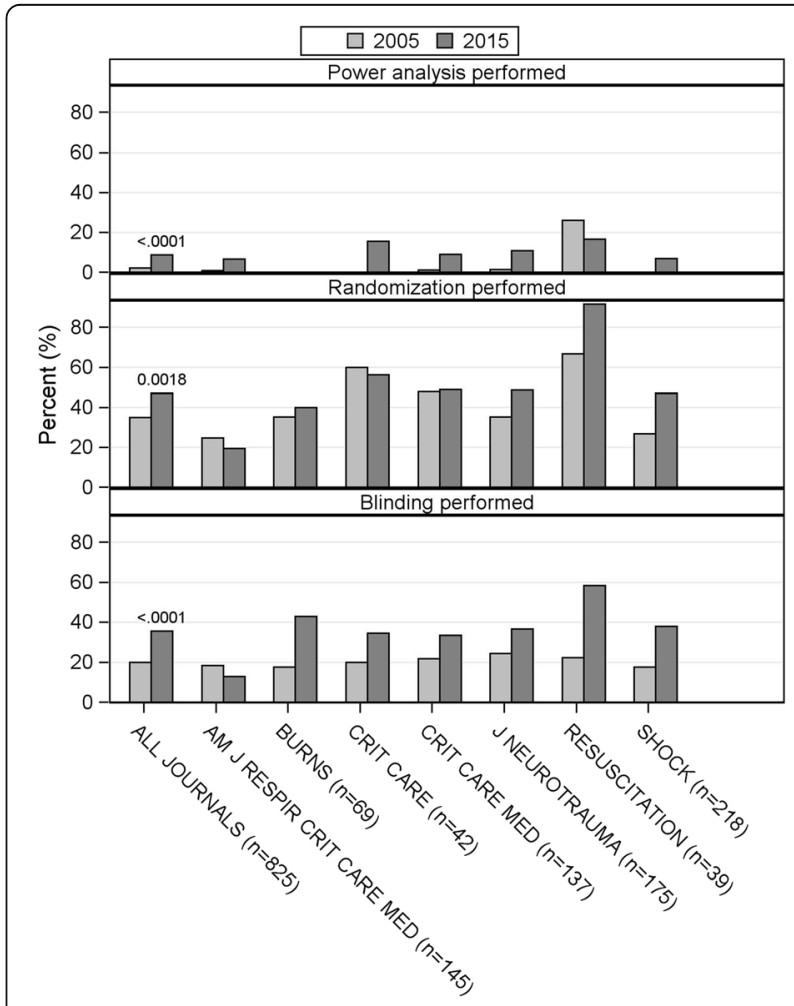

Fig. 2 Frequencies of recommended study design feature reporting per journal. Comparison was made using Chi square test 
Table 1 Reporting of recommended study design features in critical care medicine manuscripts 2005 and 2015 (binary ratings). Comparisons were made using Chi square tests ( $P$-value) and simple logistic regression (odds ratio)

\begin{tabular}{|c|c|c|c|c|c|}
\hline Study design feature & Total $(n=825)$ & $2005(n=482)$ & $2015(n=343)$ & $\begin{array}{l}\text { Bonferroni-adjusted } \\
P \text {-value }\end{array}$ & Odds Ratio $(98.3 \% \mathrm{Cl})$ \\
\hline Power analysis performed & $40(5)$ & $10(2)$ & $30(9)$ & $<0.0001$ & $4.52(1.86,11.0)$ \\
\hline Randomization performed & $330(40)$ & $169(35)$ & $161(47)$ & 0.0018 & $1.64(1.16,2.31)$ \\
\hline Blinding performed & $218(26)$ & $96(20)$ & $122(36)$ & $<0.0001$ & $2.22(1.51,3.25)$ \\
\hline
\end{tabular}

blinding 2.22 times $(1.51,3.25)$ higher in 2015 compared to 2005 (Table 1).

The highest rating of "performed and details given" was present in 2005 vs. 2015 for power analysis in $2 \%$ vs. $8 \%$, for randomization in $3 \%$ vs. $8 \%$, and for blinding in $7 \%$ vs. $13 \%$ of manuscripts. An article published in 2015 was $3.26(1.61,6.61)$ times more likely to have a higher level of reporting of power analyses than in 2005. 2015 articles were $1.67(1.21,2.32)$ times more likely to have a higher level of reporting of randomization than in 2005, and the odds of a higher level of reporting of blinding was $2.10(1.45,3.04)$ times greater in 2015 compared to 2005 (Table 2).

For the binary ratings, observed agreement between the two investigators for the 82 articles assessed was $0.95,0.93$, and 0.90 for power, randomization, and blinding respectively. Cohen's Kappa values indicated moderate agreement for power, almost perfect agreement for randomization, and substantial agreement for blinding. Gwet's $\mathrm{AC} 1$ values indicated almost perfect agreement beyond that which occurs by chance alone (Table 3). Observed agreement between the two investigators in identifying all articles reporting animal experimental research from two randomly selected journals for inclusion/exclusion in this study was 0.99 . The kappa coefficient indicates almost perfect agreement beyond that which occurs by chance alone $(0.97$ (95\% CI 0.94,0.99)).

\section{Discussion}

The quality of research and reporting of animal studies in critical care medicine journals is an area of increased interest, especially as reproducibility and successful translation of basic science results to clinical application has been low [22-24]. In addition to impeding progress in the development of novel therapies, these issues also present ethical concerns [9, 25-27]. In attempts to improve animal research quality, initiatives such as the ARRIVE guidelines have been created to improve the methodological rigor and to enhance translation [8]. To date, there are few studies examining the reporting of recommended experimental design feature to increase

Table 2 Reporting of recommended study design features in critical care medicine manuscripts 2005 and 2015 (ordinal ratings). Comparisons were made between 2005 and 2015 using Wilcoxon Rank Sum test (P-value) and proportional odds models were used to calculate odds

\begin{tabular}{|c|c|c|c|c|c|}
\hline Study design feature & Total $(n=825)$ & $2005(n=482)$ & $2015(n=343)$ & $\begin{array}{l}\text { Bonferroni-adjusted } \\
\text { P-value }\end{array}$ & Odds Ratio $(98.3 \% \mathrm{Cl})$ \\
\hline \multicolumn{6}{|l|}{ Power analysis } \\
\hline Not mentioned & 769 (93) & $464(96)$ & $305(89)$ & $<0.0001$ & $3.26(1.61,6.61)$ \\
\hline Mentioned but not performed & $16(2)$ & $8(2)$ & $8(2)$ & & \\
\hline Performed but no details given & $6(1)$ & $2(0)$ & $4(1)$ & & \\
\hline Performed and details given & $34(4)$ & $8(2)$ & $26(8)$ & & \\
\hline \multicolumn{6}{|l|}{ Randomization } \\
\hline Not mentioned & $445(54)$ & $283(59)$ & $162(47)$ & 0.0005 & $1.67(1.21,2.32)$ \\
\hline Mentioned but not performed & $50(6)$ & $30(6)$ & $20(6)$ & & \\
\hline Performed but no details given & $290(35)$ & $155(32)$ & $135(39)$ & & \\
\hline Performed and details given & $40(5)$ & $14(3)$ & $26(8)$ & & \\
\hline \multicolumn{6}{|l|}{ Blinding } \\
\hline Not mentioned & $596(72)$ & $378(78)$ & $218(64)$ & $<0.0001$ & $2.10(1.45,3.04)$ \\
\hline Mentioned but not performed & $11(1)$ & $8(2)$ & $3(1)$ & & \\
\hline Performed but no details given & $143(17)$ & $64(13)$ & $79(23)$ & & \\
\hline Performed and details given & $75(9)$ & $32(7)$ & $43(13)$ & & \\
\hline
\end{tabular}


Table 3 Inter-rater agreement for binary ratings of metrics using Cohen's Kappa, Gwet's AC1, and observed agreement

\begin{tabular}{|c|c|c|c|c|c|}
\hline Study design feature & Cohen's Kappa & $98.3 \% \mathrm{Cl}$ & $\begin{array}{l}\text { Gwet's AC1 } \\
\text { Coefficient }\end{array}$ & $98.3 \% \mathrm{Cl}$ & $\begin{array}{l}\text { Observed } \\
\text { agreement }\end{array}$ \\
\hline Power analysis & 0.58 & $0.13,1.00$ & 0.94 & $0.88,1.00$ & 0.95 \\
\hline Randomization & 0.85 & $0.72,0.99$ & 0.85 & $0.72,0.99$ & 0.93 \\
\hline Blinding & 0.79 & $0.62,0.95$ & 0.82 & $0.67,0.97$ & 0.90 \\
\hline
\end{tabular}

scientific rigor and reduce bias in animal experimental critical care research.

In our study, we evaluated the methodological quality of animal research in critical care journals in 2005 and 2015 and found a significant increase in the reporting of power analyses, randomization, and sample size calculations. Our hypothesis that these metrics are more commonly reported in 2015 compared to 2005 was confirmed. Introduced in 2010, the ARRIVE guidelines [8] may have been one of several factors that led to the improved reporting of recommended study design features in 2015. Our analysis using an ordinal scoring system still found the lowest rating category to be the most common one for every criterion assessed, even in 2015. Contemporary research in the field of critical care reports on recommended procedures to improve experimental design rigor only in a minority of manuscripts. This is in line with the limited published literature on this topic. Bara et al. [13], reviewed 77 animal research articles published in critical care journals over a six-month period in 2012 . They found that $61 \%$ reported randomization and $6 \%$ of these reported some type of allocation concealment and only $2 \%$ reported a method of randomization.

Huet et al. [12] highlighted the importance on enhancing animal research quality including improving the use of the 3Rs (replacement, reduction, refinement), which are the guiding principles for ethical animal testing [2830]. They emphasized, however, that there continues to be poor adherence to these recommendations. Festing et al. [3], emphasized the historical significance of animal research and the major contributions resulting from it: animal research has led to the advancement of immunization medicine, use of vitamins in almost eliminating diseases such as scurvy and rickets, and the discovery of insulin and its effect on metabolic diseases. Yet, they also identified a lack of adherence to good practices of research design as a major impediment to progress in medicine.

Although enhanced translation is the ultimate goal of measures to improve experimental design rigor, it remains to be determined if there has been an improvement in reproducibility or successful translation of animal experimental research results. Given the significant time lag between the description of basic science results and publication of clinical trial results, proof of a direct relationship between reported experimental design rigor and translation to novel therapies for critically ill patients will be challenging. It is also possible that some articles may not have described quality metrics that were in fact utilized in the research protocol. In addition, editors and reviewers may have recommended reporting according to the more recent ARRIVE [8] guidelines during the review process. The observed difference between 2005 and 2015 may, therefore, reflect more a change in reporting as opposed to a change in experimental practices. Of note, an innovative online tool, the "Experimental Design Assistant" was introduced in October 2015 as a guide for researchers to assist in the rigorous design of experiments [31]. However, none of the articles included in our study mentioned utilizing this resource. Further, our search strategy may not have detected all animal research articles in critical care journals in the two time periods examined. However, almost perfect agreement existed between two independent investigators in this regard. Critical care relevant research is published in other (non-critical care medicine specific) journals, and we did not include non-critical care journals in this study. Indeed, when comparing 2005 to 2015 , the annual number of animal experimental manuscripts published in critical care journals decreased by 139 articles. This contrasts with findings that overall, publications in the medical literature have been increasing in the last decade [32, 33]. Finally, publication bias was not assessed in this study. Publication bias likely has a significant impact on the quality of animal research and its ability to be translated into successful clinical trials $[34,35]$.

\section{Conclusions}

The application and reporting of recommended quality metrics in animal experimental research published in critical care medicine journals continue to be modest. However, the increase in reported measures aimed to improve experimental design quality and reduce sources of bias in 2015 compared to 2005 is promising. Reporting of blinding, randomization, and sample size estimates should be encouraged in future animal experimental publications in critical care medicine. The routine justification for the presence or absence of these study design features should be considered in reports on animal experimental research. 


\section{Abbreviations}

ARRIVE: Animal Research: Reporting of In Vivo Experiments quidelines: $\mathrm{NIH}$ : National Institutes of Health

\section{Funding}

This work was supported by the National Institutes of Health Award Number K23DA040923 to Karsten Bartels. In addition this work was supported by NIH Award Number UL1TR001082. The content of this report is solely the responsibility of the authors and does not necessarily represent the official views of the $\mathrm{NIH}$. The $\mathrm{NIH}$ had no involvement in study design, collection, analysis, interpretation of data, writing of the report, or the decision to submit the article for publication.

\section{Availability of data and materials}

The datasets used and analyzed during the current study are available from the corresponding author on reasonable request.

\section{Authors' contributions}

JSM wrote the manuscript. JSM and JMH contributed to the conduct of the study and data collection. JSM, JMH, and KB contributed to the conduct of the study. KB and AFM contributed to the study design. AFM, SKMG, and KB contributed to data analysis. JB, LMM, AFB, and SKMG played major roles interpreting the data. All authors (JSM, JMH, AFM, JB, LMM, AFB, SKMG, and $\mathrm{KB}$ ) critically revised the manuscript. $\mathrm{KB}$ secured funding for the study and performed manuscript preparation. All authors approved the final version.

\section{Ethics approval and consent to participate}

Not applicable. This was an observational bibliometric study.

\section{Consent for publication}

Not applicable

\section{Competing interests}

The authors declare that they have no competing interests.

\section{Publisher's Note}

Springer Nature remains neutral with regard to jurisdictional claims in published maps and institutional affiliations.

\section{Author details}

'Department of Anesthesiology, Medicine, and Surgery, University of Colorado, School of Medicine, Anschutz Medical Campus, 12401 E. 17th Ave., Leprino Office Building, 7th Floor, MS B-113, Aurora, CO 80045, USA. ${ }^{2}$ Adult and Child Center for Health Outcomes and Delivery Science, University of Colorado, School of Medicine, Aurora, Colorado, USA. ${ }^{3}$ Department of Psychiatry, University of Colorado, School of Medicine, Aurora, Colorado, USA. ${ }^{4}$ Department of Biostatistics \& Informatics, University of Colorado, School of Public Health, Aurora, Colorado, USA.

Received: 13 December 2017 Accepted: 19 June 2018

Published online: 05 July 2018

\section{References}

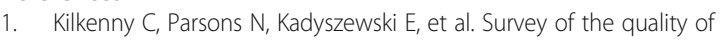
experimental design, statistical analysis and reporting of research using animals. PLoS One. 2009;4:e7824.

2. Atkinson G, Batterham AM, Dowdall N, Thompson A, Van Drongelen A From animal cage to aircraft cabin: an overview of evidence translation in jet lag research. Eur J Appl Physiol. 2014;114:2459-68.

3. Festing MF, Nevalainen T. The design and statistical analysis of animal experiments: introduction to this issue. ILAR J. 2014;55:379-82.

4. Begley CG, loannidis JP. Reproducibility in science: improving the standard for basic and preclinical research. Circ Res. 2015;116:116-26.

5. Garner JP. The significance of meaning: why do over $90 \%$ of behavioral neuroscience results fail to translate to humans, and what can we do to fix it? ILAR J. 2014:55:438-56.

6. Collins FS, Tabak LA. Policy: NIH plans to enhance reproducibility. Nature. 2014;505:612-3.

7. Galley HF. Mice, men, and medicine. Br J Anaesth. 2010;105:396-400.
8. Kilkenny C, Browne WJ, Cuthill IC, Emerson M, Altman DG. Improving bioscience research reporting: the ARRIVE guidelines for reporting animal research. PLoS Biol. 2010;8:e1000412.

9. Ferdowsian HR, Beck N. Ethical and scientific considerations regarding animal testing and research. PLoS One. 2011;6:e24059.

10. Bara M, Joffe AR. The ethical dimension in published animal research in critical care: the public face of science. Crit Care. 2014;18:R15.

11. Uhlig C, Krause H, Koch T, Gama de Abreu M, Spieth PM. Anesthesia and monitoring in small laboratory mammals used in anesthesiology, respiratory and critical care research: a systematic review on the current reporting in Top-10 impact factor ranked journals. PLoS One. 2015;10:e0134205.

12. Huet $\mathrm{O}$, de Haan JB. The ethical dimension in published animal research in critical care: the dark side of our moon. Crit Care. 2014;18:120.

13. Bara M, Joffe AR. The methodological quality of animal research in critical care: the public face of science. Ann Intensive Care. 2014:4:26.

14. von Elm E, Altman DG, Egger M, et al. The strengthening the reporting of observational studies in epidemiology (STROBE) statement: guidelines for reporting observational studies. J Clin Epidemiol. 2008;61:344-9.

15. Moher D, Liberati A, Tetzlaff J, Altman DG, Group P. Preferred reporting items for systematic reviews and meta-analyses: the PRISMA statement. Ann Intern Med. 2009;151:264-9. W64

16. Thomson Reuters, Institute for Scientific Information, National Library of Medicine (U.S.). Web of science. MEDLINE. New York: Thomson Reuters; 2016.

17. Merriam-Webster Inc. Merriam-Webster's collegiate dictionary. 11th ed. Springfield: Merriam-Webster, Inc.; 2003. p. 1623.

18. Hoerauf JM, Moss AF, Fernandez-Bustamante A, Bartels K. Study design rigor in animal-experimental research published in anesthesia journals. Anesth Analg. 2018;126:217-22.

19. Gwet KL. Computing inter-rater reliability and its variance in the presence of high agreement. Br J Math Stat Psychol. 2008;61:29-48.

20. Wongpakaran N, Wongpakaran T, Wedding D, Gwet KL. A comparison of Cohen's Kappa and Gwet's AC1 when calculating inter-rater reliability coefficients: a study conducted with personality disorder samples. BMC Med Res Methodol. 2013;13:61.

21. Landis JR, Koch GG. The measurement of observer agreement for categorical data. Biometrics. 1977:33:159-74.

22. Dyson A, Singer M. Animal models of sepsis: why does preclinical efficacy fail to translate to the clinical setting? Crit Care Med. 2009;37:S30-7.

23. Xiong Y, Mahmood A, Chopp M. Animal models of traumatic brain injury. Nat Rev Neurosci. 2013;14:128-42.

24. Reynolds PS. Twenty years after: do animal trials inform clinical resuscitation research? Resuscitation. 2012;83:16-7.

25. Hess KR. Statistical design considerations in animal studies published recently in cancer research. Cancer Res. 2011;71:625.

26. Landis SC, Amara SG, Asadullah $\mathrm{K}$, et al. A call for transparent reporting to optimize the predictive value of preclinical research. Nature. 2012;490:187-91.

27. Couzin-Frankel J. When mice mislead. Science. 2013:342:922-3. 5

28. Blache D, Martin GB, Maloney SK. Towards ethically improved animal experimentation in the study of animal reproduction. Reprod Domest Anim. 2008;43 Suppl 2:8-14.

29. Schuppli CA, Fraser D, McDonald M. Expanding the three Rs to meet new challenges in humane animal experimentation. Altern Lab Anim. 2004;32:525-32

30. Leenaars M, Savenije B, Nagtegaal A, van der Vaart L, Ritskes-Hoitinga M. Assessing the search for and implementation of the Three Rs: a survey among scientists. Altern Lab Anim. 2009;37:297-303.

31. Percie du Sert N, Bamsey I, Bate ST, et al. The experimental design assistant. PLoS Biol. 2017:15:e2003779.

32. Druss BG, Marcus SC. Growth and decentralization of the medical literature: implications for evidence-based medicine. J Med Libr Assoc. 2005:93:499-501.

33. Larsen PO, von Ins M. The rate of growth in scientific publication and the decline in coverage provided by Science Citation Index. Scientometrics. 2010;84:575-603.

34. Sena ES, van der Worp HB, Bath PM, Howells DW, Macleod MR. Publication bias in reports of animal stroke studies leads to major overstatement of efficacy. PLoS Biol. 2010;8:e1000344

35. ter Riet $\mathrm{G}$, Korevaar DA, Leenaars $\mathrm{M}$, et al. Publication bias in laboratory animal research: a survey on magnitude, drivers, consequences and potential solutions. PLoS One. 2012;7:e43404. 